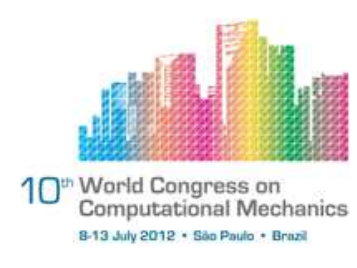

\title{
Godunov SPH with an operator-splitting procedure for materials with strength
}

\author{
A. Connolly ${ }^{1}$ and L. Iannucci ${ }^{1}$ \\ ${ }^{1}$ Department of Aeronautics, Imperial College London, London, SW7 2AZ \\ (a.connolly08@imperial.ac.uk)
}

\begin{abstract}
This paper presents a modification of the Godunov Smoothed Particle Hydrodynamics (GSPH) method of Parshikov et al. for isotropic materials with strength. The motivation behind this modification is that it facilitates a higher-order reconstruction of the left and right hand Riemann states, thus increasing the accuracy of the method. A consequence of this modification is that different smoothing kernel functions may be used within each timestep, which may be exploited to help alleviate the intrinsic instabilities of the SPH method. The paper begins with a description of the SPH method then reviews the different Godunov SPH formulations available. The modified GSPH procedure is then detailed and results are presented for one and two-dimensional test cases and compared with predictions made with the standard Artificial Viscosity SPH (AVSPH) formulation.
\end{abstract}

Keywords: SPH, Smoothed particle hydrodynamics, high velocity impact

\section{INTRODUCTION}

Smoothed particle hydrodynamics (SPH) is a Lagrangian mesh-free particle method which is particularly suitable for simulations involving large deformations. It was originally invented in the 1977 for simulating astrodynamical problems [4] and has since been applied to general fluid and solid dynamics. In solid dynamics the SPH method is most commonly used for simulations involving high-velocity impacts or other problems involving extreme material distortions. High velocity impacts create shock-waves and in a continuum level numerical scheme the spatial distretization is typically several orders of magnitude larger than the shockwave thickness [10]. Therefore, in order to maintain a stable solution, the shock-wave must be smeared across several grid-cells (or smoothed particles) such that there is a smooth variation in field quantities across the shock. In the SPH method, the resolution of a discontinuity is always limited by the smoothing kernel radius so shocks are always resolved less sharply than in grid-based methods. Traditionally in the SPH method the shock is smeared by applying an Artificial Viscosity (AV) [11] term to the momentum and energy equations. Several forms of AV have been described in the literature $[6,11,1]$ with the most common implementation (found in most SPH codes) being that of Monaghan et al. [11]. When using the artificial viscosity, without special treatments, care must be taken not to introduce excessive smoothing into smooth regions away from the shock. This may be achieved by a time-consuming trialand-error analysis [15] which may be very undesirable for the user. The special treatments 
mentioned rely on using higher-order terms $[14,2]$ to detect shock-indicative flow convergence before it occurs or an estimation of the vorticity to minimize damping in regions of pure shear [1] so locally varying damping can be applied more optimally. While these treatments reduce the undesirable effects of the $\mathrm{AV}$, they still require user-defined damping parameters which, in some cases such as hypervelocity impacts, may provide insufficient damping and cause numerical oscillations to destroy the solution. Recently, the Godunov reformulation of the SPH equations has been presented $[8,13]$ whereby the Riemann problem is solved at the mid-point between two interacting particles. The solution of the Riemann problem in Lagrangian coordinates provides the post-shock pressure and velocity at the interface between two interacting particles, equivalent to the numerical flux between two adjacent cells in a grid-based method, which automatically introduces sufficient numerical dissipation for stable integration. The advantage of these Godunov reformulations is that no user-defined damping parameters or associated sensitivity analyses are required; sufficient damping is automatically introduced into the solution. The strictly conservative GSPH method presented by Inutsuka (2002) [8] was developed for inviscid fluid simulations and maintains secondorder spatial accuracy in smooth regions by the use of a variable extrapolation procedure. The non-conservative GSPH method of Parshikov et al. (2002) [13] was developed for fluid and solid dynamics and is at most first-order accurate everywhere in the solution. The motivation behind this work was to facilitate the extension of the GSPH method of Parshikov et al. , for solid dynamics, to second-order accuracy using some of the techniques described in [8]. This is done by splitting the integration procedure into a hydrodynamic and a deviatoric step, thereby removing the complications caused by the material strength, which was first described in [7] for a free-Lagrange Voronoi tesselation method. A consequence of this modification is that allows the use of different smoothing kernel functions within the temporal discretization, which may help to alleviate the instabilities intrinsic to the SPH method [16]. The SPH method and its Godunov reformulation are described in the next section. The modification to the GSPH method is then described and some one and two dimensional results are presented and compared with standard AVSPH.

\section{THE SPH METHOD}

The basis of the SPH method is in the approximation of a function of spatial coordinates $f(\mathbf{x})$ through the approximate kernel interpolation of the function at locations surrounding the point of interest. The usual derivation [10] is to start with the identity

$$
f(\mathbf{x})=\int f\left(\mathbf{x}^{\prime}\right) \delta\left(\mathbf{x}-\mathbf{x}^{\prime}\right) d \mathbf{x}^{\prime}
$$

The delta function is replaced by some smoothing (or "kernel") function $W\left(\mathbf{x}-\mathbf{x}^{\prime}, h\right)$ with the same property as the delta function as the smoothing length $h$ tends to zero:

$$
\lim _{h \rightarrow 0} W\left(\mathbf{x}-\mathbf{x}^{\prime}, h\right)=\delta\left(\mathbf{x}-\mathbf{x}^{\prime}\right) .
$$

This gives the SPH approximation

$$
f(\mathbf{x}) \approx \int f\left(\mathbf{x}^{\prime}\right) W\left(\mathbf{x}-\mathbf{x}^{\prime}, h\right)
$$


The kernel function should satisfy the unity condition ${ }^{1}$

$$
\int W\left(\mathbf{x}-\mathbf{x}^{\prime}, h\right) d \mathbf{x}^{\prime}=1
$$

and, in order to be computationally tractable, should be compact such that

$$
W\left(\mathbf{x}-\mathbf{x}^{\prime}, h\right)=0 \quad \text { if } \quad\left|\mathbf{x}-\mathbf{x}^{\prime}\right| \geq \kappa h
$$

where $\kappa$ is some characteristic width. The smoothing convolution (3) is discretized using the mid-point rule to give

$$
f(\mathbf{x}) \approx \sum_{j} f\left(\mathbf{x}_{j}\right) W\left(\mathbf{x}-\mathbf{x}_{j}, h\right) \Delta V_{j}
$$

where $V_{j}=m_{j} / \rho_{j}$ is the volume of the smoothed particle $j$. If the function is taken as the density $\rho$, the SPH summation approximation of the density is obtained:

$$
\rho(\mathbf{x}) \approx \sum_{j} m_{j} W\left(\mathbf{x}-\mathbf{x}_{j}, h\right)
$$

It is clear from equation (7) that the kernel function should satisfy some physically intuitive properties, such as being non-negative, and monotonically decreasing as $h \rightarrow 0$. For this reason, a Gaussian, or Gaussian-like function is commonly chosen as the kernel. The derivative of a function may be obtained by using integration by parts and the divergence theorem to give

$$
\nabla \cdot f(\mathbf{x}) \approx \int_{S} f\left(\mathbf{x}^{\prime}\right) W\left(\mathbf{x}-\mathbf{x}^{\prime}, h\right) \cdot \mathbf{n} d S-\int_{\Omega} f\left(\mathbf{x}^{\prime}\right) \cdot \nabla W\left(\mathbf{x}-\mathbf{x}^{\prime}, h\right) d \mathbf{x}^{\prime} .
$$

In general, the surface integral in equation (8) is neglected in the actual computation, as it vanishes if the kernel support does not intersect the boundary of the material domain. For simulations involving free-surfaces, the neglect of the surface integral contributes to the boundary deficiency in the SPH method. As the continuum equations of motion are first-order, equation (8) may be used to formulate the equations in the SPH approximation.

\section{SPH APPROXIMATION OF EQUATIONS OF MOTION}

We will consider first the Euler equations for an inviscid fluid:

$$
\begin{aligned}
& \frac{D \rho}{D t}=-\rho \nabla \cdot \mathbf{v} \\
& \frac{D \mathbf{v}}{D t}=-\frac{1}{\rho} \nabla P \\
& \frac{D e}{D t}=-\frac{P}{\rho} \nabla \cdot \mathbf{v} .
\end{aligned}
$$

The SPH approximation of the density field may be obtained by using the summation approximation of the density (7) or by applying the identity in (8) to the continuity equation to give the smoothed approximation

$$
\frac{D \rho_{i}}{D t} \approx \rho_{i} \sum_{j} \frac{m_{j}}{\rho_{j}}\left(\dot{\mathbf{x}}_{i}-\dot{\mathbf{x}}_{j}\right) \cdot \nabla_{i} W_{i j}
$$

\footnotetext{
${ }^{1}$ The unity condition is rarely achieved in practical SPH simulations where particles rapidly become disordered.
} 
where

$$
\nabla_{i} W_{i j}=\frac{\partial}{\partial \mathbf{x}_{i}} W\left(\mathbf{x}_{i}-\mathbf{x}_{j}, h\right)
$$

and the $D / D t$ means the substantial derivative. In the SPH equations of motion derived by Inutsuka in [8], equation (7) is used for the density evolution and the identity

$$
\sum_{j} \frac{m_{j}}{\rho(\mathbf{x})} W\left(\mathbf{x}-\mathbf{x}_{j}, h\right)=1
$$

is used to derive the SPH approximation of function $f(\mathbf{x})$ which is different from (3), by assuming that $\mathbf{x} \neq \mathbf{x}_{i}$, as follows:

$$
f\left(\mathbf{x}_{i}\right) \approx \int \sum_{j} m_{j} \frac{f\left(\mathbf{x}^{\prime}\right)}{\rho\left(\mathbf{x}^{\prime}\right)} W\left(\mathbf{x}^{\prime}-\mathbf{x}_{i}, h\right) W\left(\mathbf{x}^{\prime}-\mathbf{x}_{j}, h\right) d \mathbf{x}^{\prime} .
$$

Inutsuka [8] notes that the standard SPH approximation for a function follows from (13) if one assumes that $W\left(\mathbf{x}_{j}-\mathbf{x}^{\prime}, h\right)=\delta\left(\mathbf{x}^{\prime}-\mathbf{x}_{j}\right)$. Inutsuka's formulation for the SPH approximation of the momentum and energy equations derive from (13) and, stated, they take the form:

$$
\begin{aligned}
& \frac{D \dot{\mathbf{x}}_{i}}{D t} \approx-\sum_{j} \int \frac{P(\mathbf{x})}{\rho^{2}(\mathbf{x})}\left[\frac{\partial}{\partial \mathbf{x}_{i}}-\frac{\partial}{\partial \mathbf{x}_{j}}\right] W\left(\mathbf{x}-\mathbf{x}_{i}, h\right) W\left(\mathbf{x}-\mathbf{x}_{j}, h\right) d \mathbf{x} \\
& \frac{D e_{i}}{D t} \approx-\sum_{j} \int \frac{P(\mathbf{x})}{\rho^{2}(\mathbf{x})}\left(\mathbf{v}(\mathbf{x})-\dot{\mathbf{x}}_{i}\right) \cdot\left[\frac{\partial}{\partial \mathbf{x}_{i}}-\frac{\partial}{\partial \mathbf{x}_{j}}\right] W\left(\mathbf{x}-\mathbf{x}_{i}, h\right) W\left(\mathbf{x}-\mathbf{x}_{j}, h\right) d \mathbf{x}
\end{aligned}
$$

where the term $\mathbf{v}(\mathbf{x})$ is the fluid velocity at $\mathbf{x}$ and the term $\dot{\mathbf{x}}_{i}$ is the smoothed velocity at $\mathbf{x}_{i}$, which Inutsuka [8] shows has an error of $\mathcal{O}\left(h^{2}\right)$. The integrals in (14) are analytically evaluated by using the property of the Gaussian kernel function and an interpolation of $1 / \rho^{2}(\mathbf{x})$ around $\mathbf{x}_{i}$ and $\mathbf{x}_{j}$ to give

$$
\begin{aligned}
& \frac{D \dot{\mathbf{x}}_{i}}{D t} \approx-2 \sum_{j} m_{j} P_{i j}^{*} V_{i j}^{2}(h) \nabla_{i} W\left(\mathbf{x}_{i}-\mathbf{x}_{j}, \sqrt{2} h\right) \\
& \frac{D e_{i}}{D t} \approx-2 \sum_{j} m_{j} P_{i j}^{*} V_{i j}^{2}(h)\left(\mathbf{v}_{i j}^{*}-\dot{\mathbf{x}}_{i}\right) \cdot \nabla_{i} W\left(\mathbf{x}_{i}-\mathbf{x}_{j}, \sqrt{2} h\right) .
\end{aligned}
$$

The star superscripts in (15) indicate the interpolated quantities at some point along the line joining the particles $\mathbf{x}_{i}$ and $\mathbf{x}_{j}$ and the term $V_{i j}^{2}(h)$ and the $\sqrt{2}$ coefficient on the smoothing length follow directly from the interpolation procedure. A detailed derivation of this is given in [8].

In contrast to the method of Inutsuka [8], the SPH Euler equations of Parshikov et al. [13] are derived in a straightforward manner using the SPH approximation of the derivative of a function (8) and the assumption that $W\left(\mathbf{x}_{j}-\mathbf{x}^{\prime}, h\right)=\delta\left(\mathbf{x}^{\prime}-\mathbf{x}_{j}\right)$ in (13), giving

$$
\begin{aligned}
\frac{D \rho_{i}}{D t} & \approx \rho_{i} \sum_{j} \frac{m_{j}}{\rho_{j}}\left(\dot{\mathbf{x}}_{i}-\dot{\mathbf{x}}_{j}\right) \cdot \nabla_{i} W_{i j} \\
\frac{D \dot{\mathbf{x}}_{i}}{D t} & \approx-\frac{1}{\rho_{i}} \sum_{j} \frac{m_{j}}{\rho_{j}}\left(P_{i}+P_{j}\right) \nabla_{i} W_{i j} \\
\frac{D e_{i}}{D t} & \approx \frac{1}{2 \rho_{i}} \sum_{j} \frac{m_{j}}{\rho_{j}}\left(P_{i}+P_{j}\right)\left(\dot{\mathbf{x}}_{i}-\dot{\mathbf{x}}_{j}\right) \cdot \nabla_{i} W_{i j} .
\end{aligned}
$$


Note that the smoothed approximation of the continuity equation is used instead of the SPH summation approximation of the density (7). This is important for simulations of soliddynamics, where free surfaces are present, as the unity condition (4) is not satisfied when the kernel support intersects the boundary and thus using the SPH summation approximation for the density would lead to a significant underestimation of the density near the boundary and cause unphysical motion which is exacerbated when materials with stiff equations of state, such as solids, are present in the simulation. This unphysical motion due to kernel under-integration does not arise when using the SPH approximation of the continuity equation for the density evolution. For these reasons, the modified Godunov SPH method uses equations (16) to solve the Euler equations.

\section{GODUNOV SPH REFORMULATION}

In Parshikov et al. [13], the equations (16) rewritten by resolving the velocities along the vector $\mathbf{n}_{i j}=\mathbf{x}_{i}-\mathbf{x}_{j} /\left|\mathbf{x}_{i}-\mathbf{x}_{j}\right|$ connecting two interacting particles

$$
v_{i}^{r}=\dot{\mathbf{x}}_{i} \cdot \mathbf{n}_{i j}
$$

which is then used to make the substitution for the mean value of the resolved velocity (superscript $r$ ) at the mid-point between two interacting particles

$$
\frac{1}{2}\left(v_{i}^{r}+v_{j}^{r}\right) \rightarrow v_{i j}^{*}
$$

The same thing is done with the pressures

$$
\frac{1}{2}\left(P_{i}+P_{j}\right) \rightarrow P_{i j}^{*}
$$

Substituting $P_{i j}^{*}$ and $v_{i j}^{*}$ into (16) and rearranging then gives the modified equations as

$$
\begin{aligned}
& \frac{D \rho_{i}}{D t} \approx 2 \rho_{i} \sum_{j} \frac{m_{j}}{\rho_{j}}\left(v_{i}^{r}-v_{i j}^{*}\right) \mathbf{n}_{i j} \cdot \nabla_{i} W_{i j} \\
& \frac{D \dot{\mathbf{x}}_{i}}{D t} \approx-\frac{2}{\rho_{i}} \sum_{j} \frac{m_{j}}{\rho_{j}} P_{i j}^{*} \nabla_{i} W_{i j} \\
& \frac{D e_{i}}{D t} \approx \frac{2}{\rho_{i}} \sum_{j} \frac{m_{j}}{\rho_{j}} P_{i j}^{*}\left(v_{i}^{r}-v_{i j}^{*}\right) \mathbf{n}_{i j} \cdot \nabla_{i} W_{i j} .
\end{aligned}
$$

When $P_{i j}^{*}$ and $v_{i j}^{*}$ are the values in the star-region resulting from the solution of the Riemann problem at the mid-point of the two interacting particles, no additional dissipation terms are required in the equations (20). The left and right states of the Riemann problem correspond to the field values at the $\mathbf{x}_{j}$ and $\mathbf{x}_{i}$ positions respectively. When considering an ideal gas, the Riemann solution may be solved exactly, and inexpensively, within a few iterations using the exact Riemann solver of van Leer (1979) [17]. However, when considering solids with complex equations of state an exact iterative solution is prohibitively expensive and an approximate non-iterative Riemann solver may be used instead, such as the two-shock solver of Dukowicz (1985) [3]. In the GSPH equations of Inutsuka (15), the $P_{i j}^{*}$ and $v_{i j}^{*}$ are replaced directly by the Riemann solutions in the star-region. This type of solution procedure corresponds to a spatially first-order Godunov method. 


\section{HIGHER-ORDER RECONSTRUCTION}

In the GSPH method of Inutsuka [8] the left and right hand states of the Riemann problem are linearly reconstructed. Using the SPH approximation of the gradient;

$$
\nabla f\left(\mathbf{x}_{i}\right) \approx \sum_{j} \frac{m_{j}}{\rho_{j}}\left(f\left(\mathbf{x}_{j}\right)-f\left(\mathbf{x}_{i}\right)\right) \nabla_{i} W_{i j}
$$

and taking into account the domain of influence, the reconstruction of the left and right hand Riemann states is written as

$$
\begin{aligned}
& f_{R}=f\left(\mathbf{x}_{i}\right)-\frac{r_{i j}}{2} \nabla f\left(\mathbf{x}_{i}\right) \cdot \mathbf{n}_{i j}\left[1-c_{i} \Delta t\right] \\
& f_{L}=f\left(\mathbf{x}_{j}\right)+\frac{r_{i j}}{2} \nabla f\left(\mathbf{x}_{j}\right) \cdot \mathbf{n}_{i j}\left[1-c_{j} \Delta t\right]
\end{aligned}
$$

where $r_{i j}=\left|\mathbf{x}_{i}-\mathbf{x}_{j}\right|, c$ is the sound speed at the particle's position and $\Delta t$ is the current time-step. In order to satisfy the monotonicity constraint at the shock [5], the gradients must be modified such that the first-order method is recovered. In [8] this is done by setting the gradients to zero when the interacting particles are approaching each other at a velocity close to the minimum wave speed of both interacting particles. A technique to automatically fulfil the monotonicity constraint by modifying the local gradient based on the harmonic mean of the gradients of adjacent grid-cells is given by van Leer (1979) [17]. A technique to apply this type of slope-limiting in the case of an unstructured grid defined by pair-wise connectivity, as in SPH, is presented in [12] and makes use of the difference gradient and the SPH smoothed approximation of the gradient. The equivalent of the local gradient at three adjacent gridcells is defined by the projecting the SPH approximation of the gradient along the vector of connectivity. From [12], the gradient between particles $i$ and $j$ is defined as

$$
\Delta Q_{1}=\frac{Q_{j}-Q_{i}}{r_{i j}}
$$

where $Q$ represents the field quantity under consideration. The quantity at the "projected particle" $j_{\text {proj }}$ is estimated using the SPH approximation of the gradient at particle $i$ using

$$
Q_{j_{p r o j}}=Q_{i}+r_{i j} \nabla Q_{i} \cdot \mathbf{n}_{i j}
$$

and the gradient between particle $i$ and the projected particle $j_{\text {proj }}$ is evaluated in the same way as in equation (23)

$$
\Delta Q_{2}=\frac{Q_{j_{p r o j}}-Q_{i}}{r_{i j}}=\nabla Q_{i} \cdot \mathbf{n}_{i j}
$$

The van Leer limited harmonic average for of the gradient around particle $i$ is defined as

$$
\nabla f\left(\mathbf{x}_{i}\right)_{m o n}=\left\{\begin{array}{lll}
\frac{2 \Delta Q_{1} \Delta Q_{2}}{\Delta Q_{1}+\Delta Q_{2}} & \text { if } & \Delta Q_{1} \Delta Q_{2}>0 \\
0 & \text { if } & \Delta Q_{1} \Delta Q_{2}<0 .
\end{array}\right.
$$

The same procedure is applied to particle $j$ using a projected particle $i_{\text {proj }}$. The result of this slope limiting procedure is shown schematically in Figure 1. 


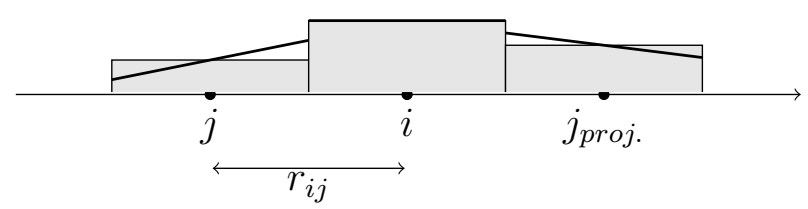

Figure 1. Schematic of the slope limiting procedure. The sign of the slope changes between particle $j$ and the projected particle $j$, therefore no reconstruction is applied to the right-hand side of the Riemann problem.

\section{TIME OPERATOR SPLITTING PROCEDURE}

In order to introduce the effect of material strength to the solution, we must consider the full Cauchy stress tensor in the equations of motion. Following the procedure described in [7], the stress tensor is decomposed into the diagonal and non-diagonal components

$$
\sigma_{i}^{\alpha \beta}=\tau_{i}^{\alpha \beta}-P_{i} \delta^{\alpha \beta}
$$

and the integration procedure operates on each component in sequence.

\subsection{Hydrodynamic stage}

The integration procedure starts by solving the GSPH Euler equations of Parshikov et al. (20) using the pressure $P_{i j}^{*}$ and velocity $\mathbf{v}_{i j}^{*}$ in the star region resulting from the solution of the Riemann problem at the mid-point between two interacting particles. In this work the two-shock approximate non-iterative Riemann solver [3] is used. The conserved quantities are then advanced by one time-step to obtain an intermediate hydrodynamic state, shown by a tilde, which is used for the deviatoric stage of the split integration procedure;

$$
\begin{aligned}
\tilde{\dot{\mathbf{x}}}_{i} & =\dot{\mathbf{x}}_{i}^{n}+\Delta t \tilde{\ddot{\mathbf{x}}}_{i} \\
\tilde{e}_{i} & =e_{i}^{n}+\Delta t \dot{e}_{i} \\
\tilde{\rho}_{i} & =\rho_{i}^{n}+\Delta t \dot{\rho}_{i} .
\end{aligned}
$$

Here the superscript $n$ denotes the value at the " $n^{t h}$ " time-step. A consequence of this timesplit integration procedure is that a different kernel function may be used for the next (deviatoric) step. If a different kernel function is to be used, the particle positions must be integrated to an intermediate location using the hydrodynamic velocity;

$$
\tilde{\mathbf{x}}_{i}=\mathbf{x}_{i}+\Delta t \tilde{\dot{\mathbf{x}}}_{i}
$$

and the derivatives of the kernel function recalculated using

$$
\nabla_{i} W_{i j}=\frac{\tilde{\mathbf{x}}_{i}-\tilde{\mathbf{x}}_{j}}{r_{i j}} \frac{\partial W_{i j}}{\partial r_{i j}},
$$

where $r_{i j}=\left|\tilde{\mathbf{x}}_{i}-\tilde{\mathbf{x}}_{j}\right|$. If the same kernel function is to be used for both the hydrodynamic and deviatoric states it is not necessary to update the particle positions to an intermediate location and recalculate the kernel derivatives. 


\subsection{Deviatoric stage}

In the deviatoric stage the Jaumann objective stress rate $\dot{\tau}_{i}$ is calculated, with the strainrates $\dot{\epsilon}_{i}$ and rotation-rates $\dot{\omega}_{i}$ obtained from the intermediate hydrodynamic velocity $\tilde{\mathbf{x}}_{i}$;

$$
\dot{\tau}_{i}^{\alpha \beta}=2 G\left(\dot{\epsilon}_{i}^{\alpha \beta}-\frac{1}{3} \delta^{\alpha \beta} \dot{\epsilon}_{i}^{\gamma \gamma}\right)+\tau_{i}^{\alpha \gamma} \dot{\omega}_{i}^{\beta \gamma}+\tau_{i}^{\gamma \beta} \dot{\omega}_{i}^{\alpha \gamma}
$$

where $G$ is the shear modulus of the material. The deviatoric stress field is then integrated to an intermediate state, shown by the tilde

$$
\tilde{\tau}_{i}=\tau_{i}^{n}+\Delta t \dot{\tau}_{i}
$$

The intermediate deviatoric stress $\tilde{\tau}_{i}$ is then used to calculate the accelerations and the rate of change of specific internal energy due to the material strength, where the average deviatoric stress $\tau_{a v}=\left(\tau_{i}+\tau_{j}\right) / 2$ is used;

$$
\begin{aligned}
& \ddot{\mathbf{x}}_{i}^{\prime} \approx \frac{1}{\rho_{i}} \sum_{j} \frac{m_{j}}{\rho_{j}} \tau_{a v} \nabla_{i} W_{i j} \\
& \dot{e}_{i}^{\prime} \approx-\frac{2}{\rho_{i}} \sum_{j} \frac{m_{j}}{\rho_{j}} \tau_{a v}\left(v_{i j}^{*}-\tilde{v}_{i}^{r}\right) \mathbf{n}_{i j} \cdot \nabla_{i} W_{i j} .
\end{aligned}
$$

These rates are then used to update the specific internal energy and particle velocities to their final states completing the time integration procedure for these quantities:

$$
\begin{gathered}
\dot{\mathbf{x}}_{i}^{n+1}=\tilde{\dot{\mathbf{x}}}_{i}+\Delta t \ddot{\mathbf{x}}_{i}^{\prime}, \\
e_{i}^{n+1}=\tilde{e}_{i}+\Delta t \dot{e}_{i}^{\prime} .
\end{gathered}
$$

Note that the intermediate hydrodynamic density is taken as the final state $\left(\rho_{i}^{n+1}=\tilde{\rho}_{i}\right)$ as the deviatoric step takes place at constant volume. The last part of the deviatoric step is to update the particle positions and stress field to their final states and apply the yield criterion. The timecentered velocity, $\dot{\mathbf{x}}_{i}^{T C}=\left(\dot{\mathbf{x}}_{i}^{n}+\dot{\mathbf{x}}_{i}^{n+1}\right) / 2$, is used to calculate the strain-rate and rotation rate and the Jaumann objective stress rate is calculated as in equation (31). The deviatoric stress is then updated as in equation (32) and the appropriate yield criterion applied. The particle positions are then updated to their final states using the time-centered velocity

$$
\mathbf{x}_{i}^{n+1}=\mathbf{x}_{i}^{n}+\Delta t \dot{\mathbf{x}}_{i}^{T C} .
$$

The smoothing length is evolved in time by linking it to the velocity divergence through the GSPH continuity equation (20), using

$$
\dot{h}_{i}=-\frac{h_{i}^{n} \dot{\rho}_{i}}{\rho_{i}^{n} d}
$$

where $d$ is the number of dimensions. The split-integration scheme described above is approximately twice as computationally expensive as the standard AVSPH method. It should be noted, however, that the stable timestep may be much greater than AVSPH [8]. 


\section{RESULTS}

Results of some one and two dimensional fluid and solid dynamics simulations are presented. In all the simulations the CFL number and smoothing length coefficient were kept fixed at 0.3 and 1.2 respectively and the smoothing kernel used was the Gaussian, unless stated otherwise.

\subsection{Shock-tube simulations}

The one-dimensional shock-tube simulation is useful to test the accuracy of the numerical method. Here no comparison is made with the standard AVSPH method as it has been extensively studied in the past. The initial conditions at either side of the discontinuity (centered at $x=0$ ) are given in Table 1. The inter-particle spacing was kept constant, corre-

$$
\begin{array}{l|l}
\rho_{l}=1.0 & \rho_{r}=0.125 \\
P_{l}=1.0 & P_{r}=0.1 \\
v_{l}=0.0 & v_{r}=0.0
\end{array}
$$

Table 1. Shock-tube initial conditions.

sponding to an $8: 1$ particle mass ratio. Simulations were performed with 100, 200 and 400 particles in the region $-0.5<x<0.5$ to show the convergence properties. The ideal-gas equation of state and an exact iterative Riemann solver [17] were used and the solution was integrated to $t=0.2$. The new integration procedure is identical to the one in Parshikov et $a l$. when the material strength is set to zero. The results of the shock-tube simulation for three different particle discretizations using first and second order reconstruction are shown in Figures $2 \mathrm{a}$ and $2 \mathrm{~b}$ respectively. The density field in the region between the rarefaction and

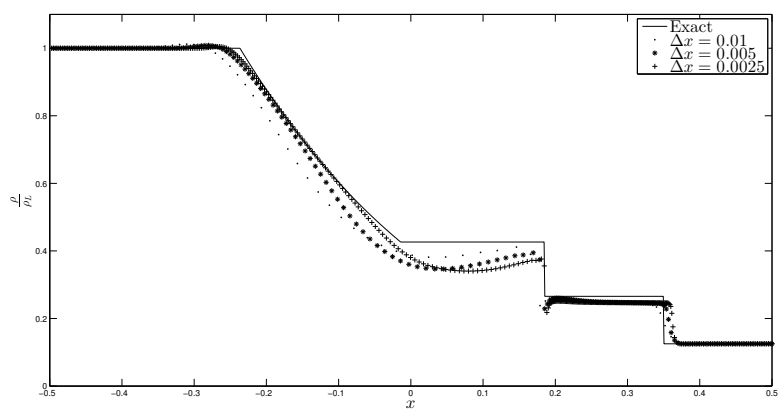

(a) $\frac{\rho}{\rho_{l}}$ with $1^{s t}$ order reconstruction.

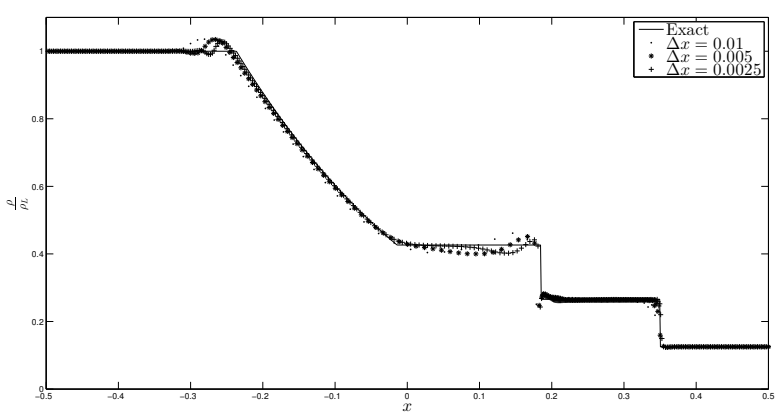

(b) $\frac{\rho}{\rho_{l}}$ with $2^{\text {nd }}$ order reconstruction.

Figure 2. Results for three different levels of spatial discretization using the GSPH method with first and second-order reconstruction of the Riemann states. The exact solution is shown by the continuous line.

the contact surface is calculated with a greater accuracy and the rarefaction is resolved more sharply with $2^{\text {nd }}$ order reconstruction. 


\subsection{Taylor-bar impact}

This problem is taken from [9] and involves a two-dimensional iron bar impacting a frictionless rigid wall (simulated by "ghost" particles reflected across the $x$-axis) at $200 \mathrm{~ms}^{-1}$. The material properties are identical to those in [9] and a Mie-Gruneisen equation of state and von-Mises yield criterion are used. The simulations are integrated to $40 \mu s$. Figure $4 \mathrm{a}$ shows the deformation using the AVSPH method with damping parameters $(a, b)=(1,1)$, and similar results are found with $(a, b)=(2,2)$. Figure $4 \mathrm{~b}$ shows the deformation using the modified GSPH method with the M4 cubic spline kernel used for both hydrodynamic and deviatoric stages of the integration procedure. Figure $4 \mathrm{c}$ shows the deformation using the modified GSPH method with the M4 cubic spline kernel used for the hydrodynamic stage and a quadratic kernel used for the deviatoric stage of the time integration procedure. The derivative of the quadratic kernel is discontinuous, however the magnitude of the derivative monotonically increases as particles approach one another, thus the compressive instability characteristic of the bell-shaped kernels does not occur. The M4 piecewise cubic spline and the quadratic kernel and their first derivatives are plotted in Figure 3.

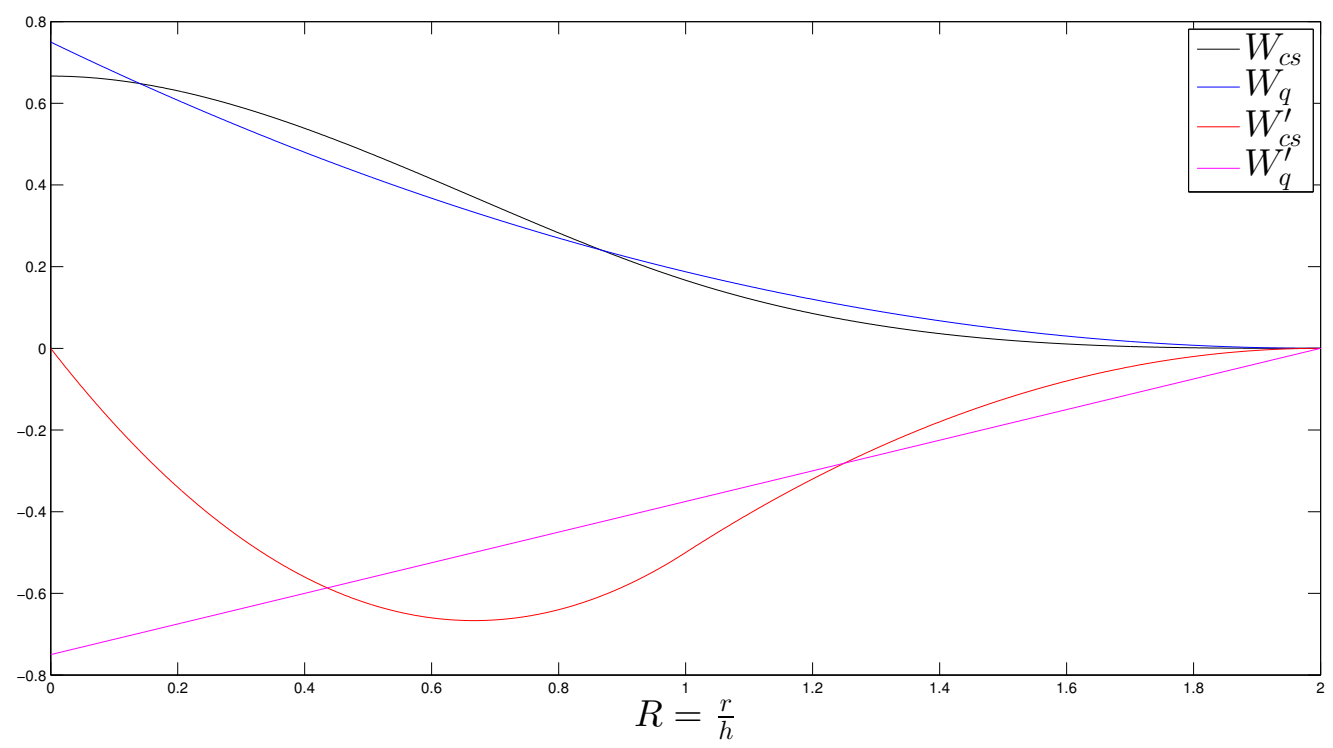

Figure 3. The values of the M4 piecewise cubic spline and quadratic kernel functions and their first derivatives.

Quite how the interpolation accuracy is affected due to the use of different kernels is not well understood - this will be investigated in future work. It is important to note that, when using different kernel functions, the kernel support radii should be equal such that the time-consuming neighbour finding algorithm does not have to be called twice. It is clear that the modified GSPH method produces significantly different results to the AVSPH method. The "mushroom" deformation close to the impact point is more confined to the impact region in the AVSPH results compared to the modified GSPH results and the pressure-field is not as smooth. With the modified GSPH method, bands of neighbouring particles do not display the pressure oscillations, as seen with the AVSPH method, and the clustering instability is less pronounced. The use of a mixed kernel formulation in Figure $4 \mathrm{c}$ results in a more tightly packed final configuration. Future work will compare experimental results with Taylor-bar 


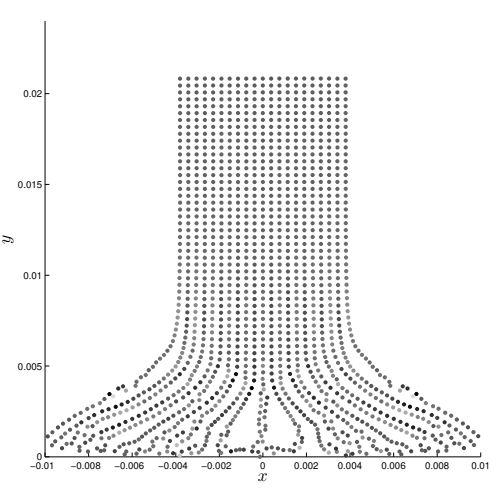

(a) AVSPH

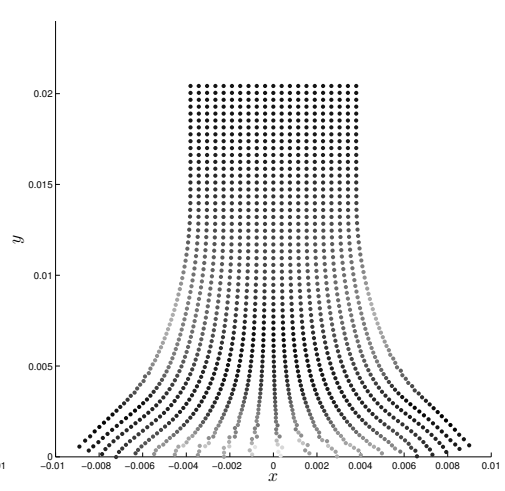

(b) GSPH fixed

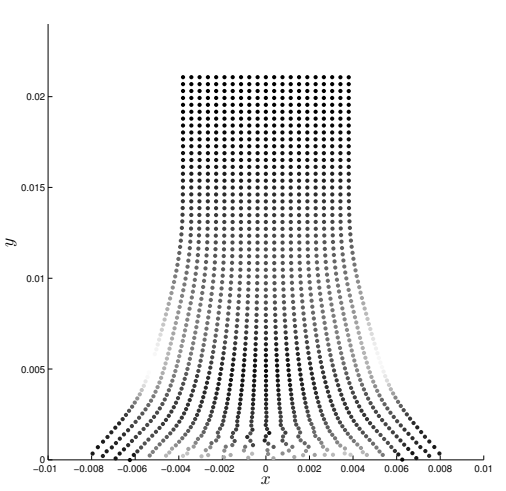

(c) GSPH mixed

Figure 4. Results of the Taylor-bar impact simulations with the standard AVSPH method with artificial viscosity damping coefficients of $(1,1)$, the first order GSPH method with fixed kernel functions and the first order GSPH method with mixed kernel functions. The gray scales indicate the pressure field and the $x$ and $y$ units are in meters.

impact simulations using a more realistic yield criterion. To compare the first and secondorder spatial reconstruction in two dimensions, the pressure field in the Taylor-bar simulations are shown at $t=2 \mu \mathrm{s}$ in Figures $5 \mathrm{a}$ and $5 \mathrm{~b}$ respectively. The use of a second-order spatial reconstruction results in a much more sharply resolved shock-front compared with the firstorder reconstruction.

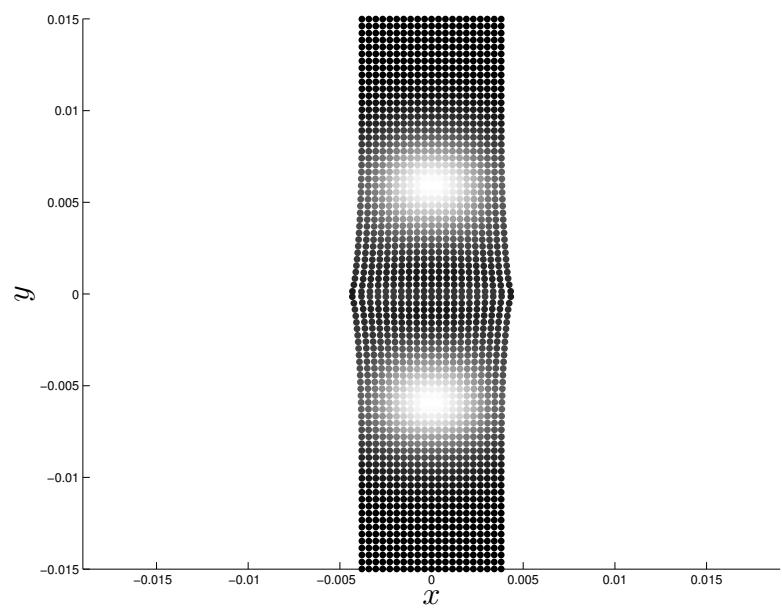

(a) GSPH $1^{\text {st }}$ order

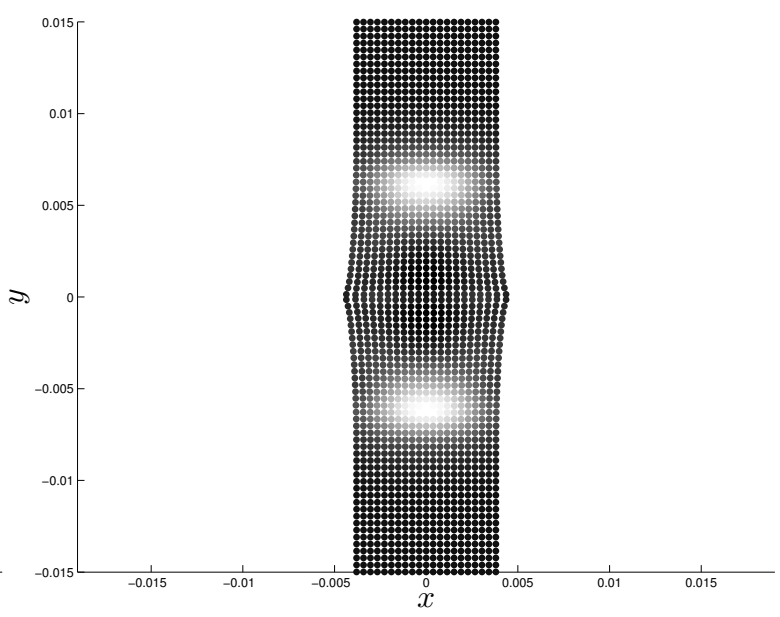

(b) GSPH $2^{\text {nd }}$ order

Figure 5. Results of the Taylor-bar impact simulations after $2 \mu s$ of integration. The gray scales indicate pressures.

\subsection{Hypervelocity impact}

This simulation is taken from [7] and involves a hypervelocity collision between a circular aluminium impactor and thin rectangular aluminium plate. The impactor travels with an initial velocity of $3.1 \mathrm{kms}^{-1}$. The solution is integrated to $8 \mu \mathrm{s}$. All material properties are the same as those in [7] and a von-Mises yield criterion and stiffened-gas equation of state 
are used. The results using the modified GSPH method (with the same kernel used for both integration stages and first-order reconstruction), the AVSPH method with $(a, b)=(1,1)$ and $(a, b)=(2,2)$ are shown in Figures 6,7 and 8 respectively. The impactor has retained

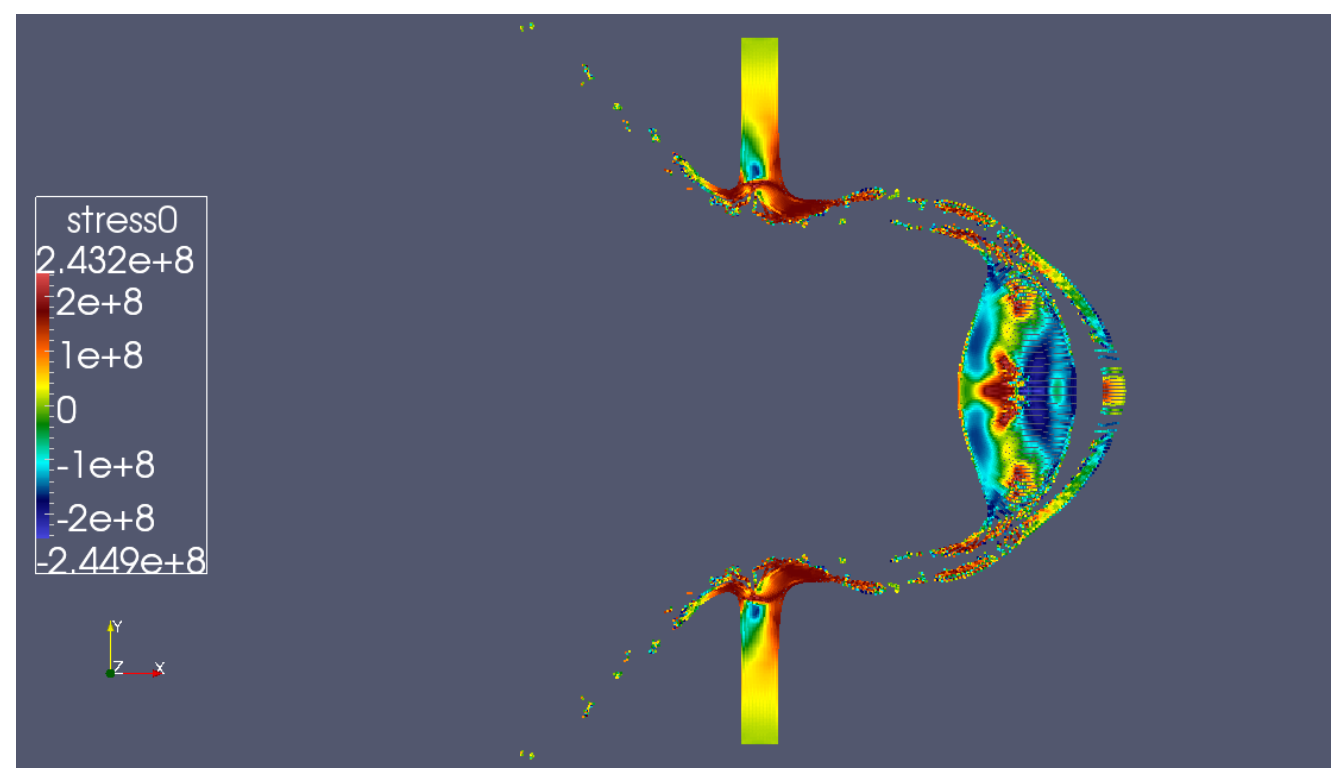

Figure 6. Hypervelocity impact results using the modified GSPH method. The colours show the $x$ direction deviatoric stresses (units are in Pascals).

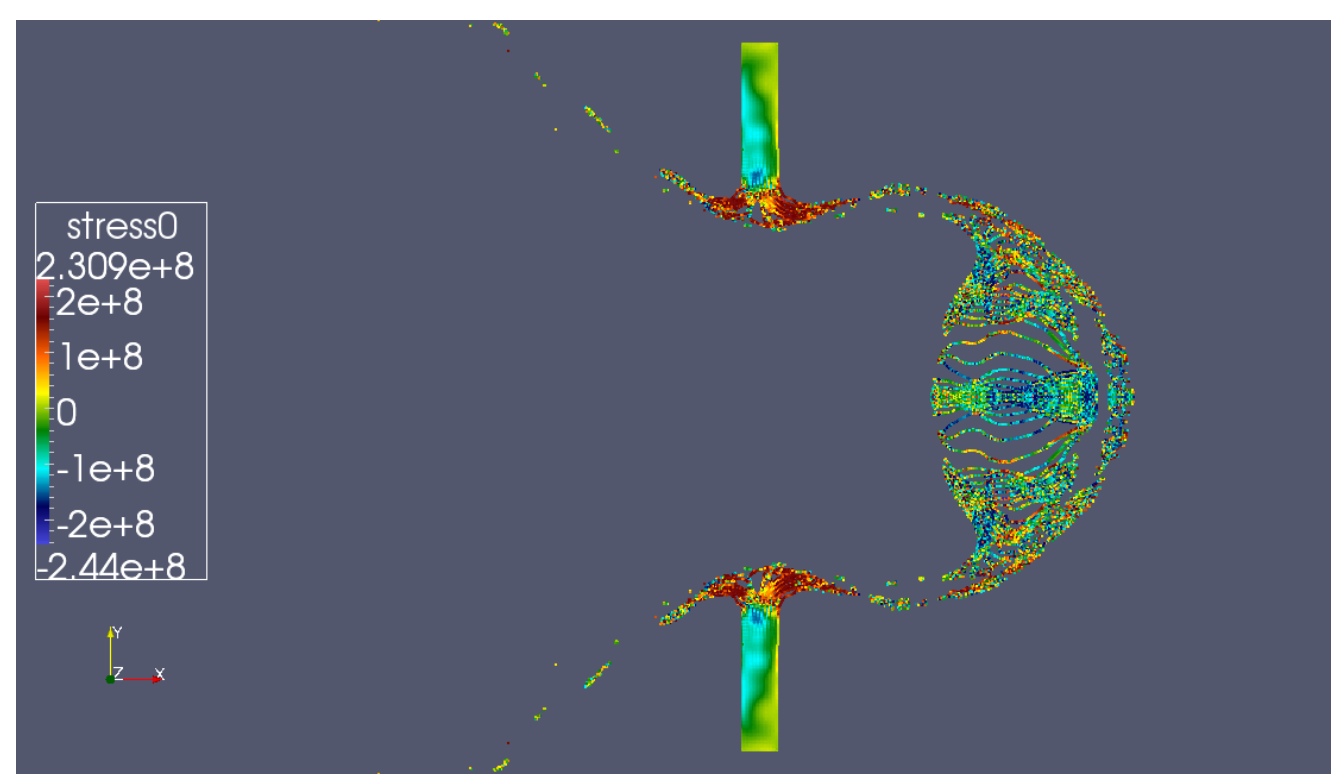

Figure 7. Hypervelocity impact results using the AV SPH method with damping parameters $(a, b)=(1,1)$. The colours show the $x$ direction deviatoric stresses (units are in Pascals).

its continuity with the modified GSPH method, whereas it has numerically fragmented with both AV SPH results due to the tensile instability. The deviatoric stress field is smooth in the modified GSPH method results compared to the AV SPH results. There are, however, regions of numerical fragmentation in the modified GSPH results as, without special treatments, the tensile instability is intrinsic to the SPH method. It should be noted that any fragmentation 


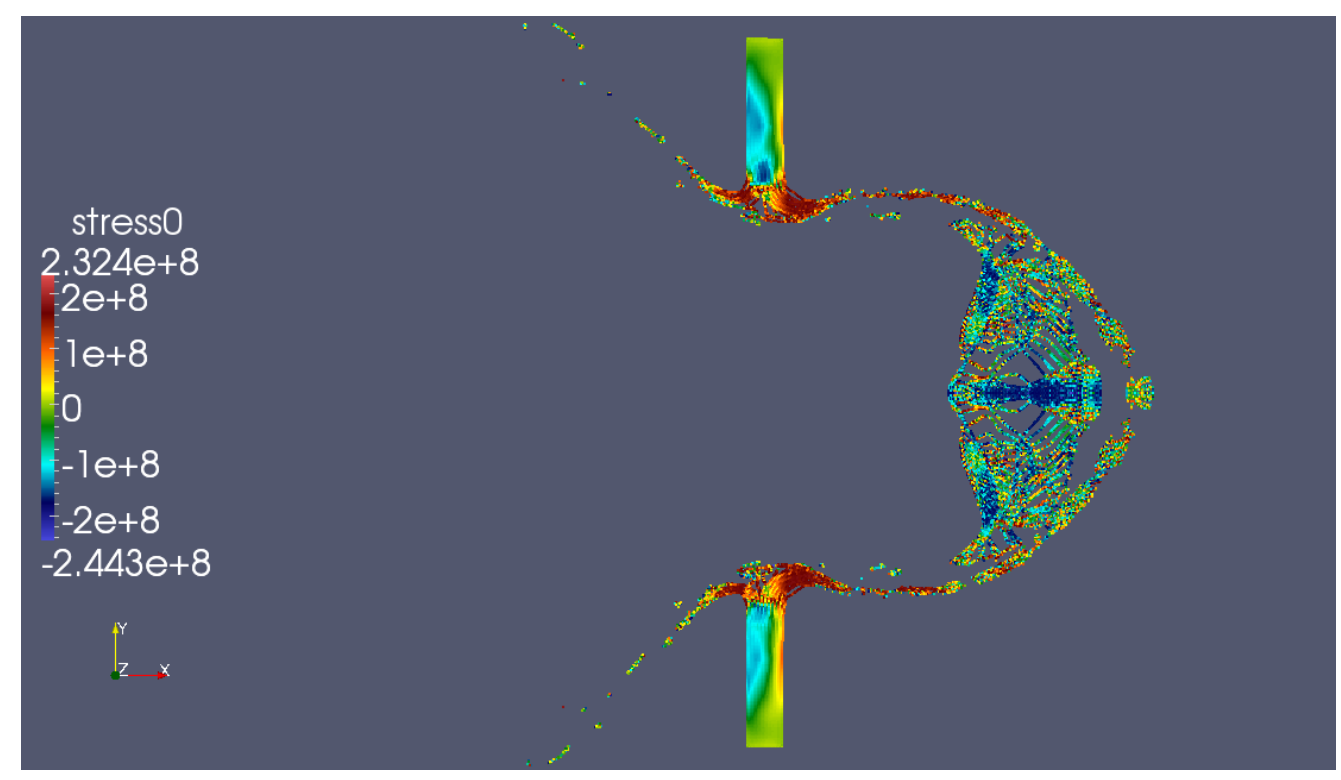

Figure 8. Hypervelocity impact results using the AV SPH method with damping parameters $(a, b)=(2,2)$. The colours show the $x$ direction deviatoric stresses (units are in Pascals).

seen in the results is unphysical as there was no failure model in the constitutive equations used.

\subsection{Shear simulation}

This simulation is similar to that in Parshikov et al. and involves the strong shearing of an initially continuous fluid material. The Mie-Gruneisen equation of state is used, with material properties equal to that used in the Taylor-bar impact simulation above. The strength is set to zero, therefore the equations being solved correspond to the Euler equations for an inviscid fluid. In the initial configuration, the particles above the $x$ axis are moving to the right at $254 \mathrm{~ms}^{-1}$. The solution is integrated to $100 \mu \mathrm{s}$. Results using the modified GSPH method with first and second-order reconstruction and the AVSPH method for $(a, b)=$ $(1,1)$ and $(a, b)=(2,2)$ are shown in Figure 9, where the grayscales represent the pressure field. As noted by in Parshikov et al. , the Godunov reformulation of the SPH equations has a high numerical viscosity, which is shown clearly by the smooth centre-line curve shown in black. This unphysical shear viscosity is substantially reduced using the second-order reconstruction method. The AVSPH results show that the numerical viscosity with the AV damping coefficients set at $(a, b)=(1,1)$ is similar to the GSPH method with second-order reconstruction.

\section{DISCUSSION AND CONCLUSIONS}

A modification to the GSPH method of Parshikov et al. [13] has been described and comparisons with the standard AVSPH have been presented. The new method utilizes a timeoperator-splitting procedure first proposed by Howell et al. [7] to negate the need for solving the Riemann problem for the transversal wave-system. The motivation behind this modification was to facilitate a higher-order spatial discretization based on a Monotone Upwind 

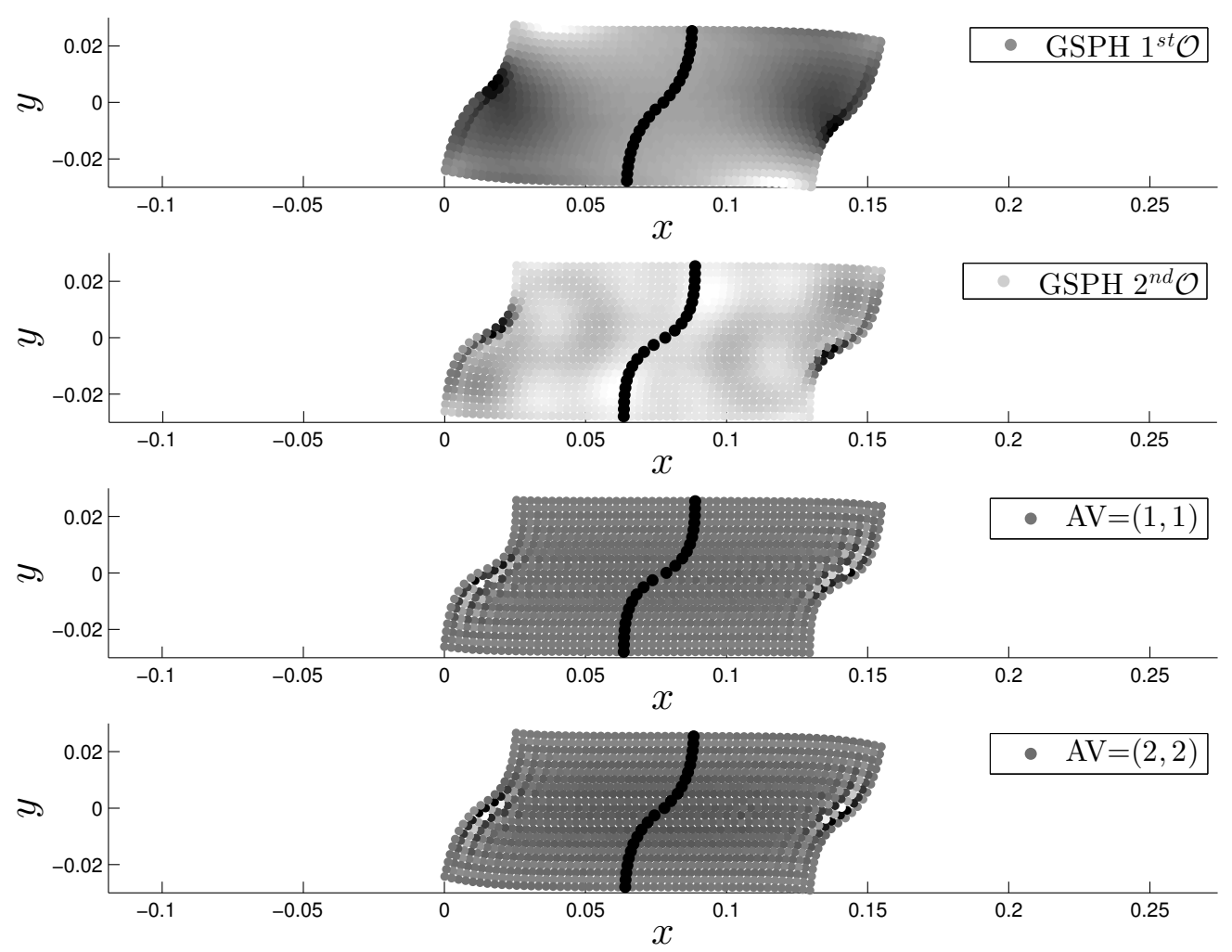

Figure 9. Comparison of the numerical viscosity of the modified GSPH methods compared to the standard AVSPH method. The initial vertical centreline of particles is shown in the final configuration in black. The grayscales indicate the pressure-field and the $x$ and $y$ units are in meters.

Scheme for Conservation Laws (MUSCL) type approach described in [8] and [12] without complication caused by the material strength. A consequence of the modification to the timeintegration procedure is that different smoothing kernels may be used within each time-step. The results for simulations involving solids show that the method compares favourably with AVSPH and the intrinsic SPH instabilities are alleviated to some degree. The method, however, is about twice as computationally expensive as the standard AVSPH method. A stability analysis, the conservation properties, the use of different kernel functions within each timestep and the investigation of different methods for higher-order reconstruction of the Riemann states will be the focus of future work.

\section{Acknowledgements}

The authors would like to thank the UK Defence Science and Technology Labs (Dstl) for sponsoring the research.

\section{References}

[1] D. S. Balsara. von-Neumann analysis of smoothed particle hydrodynamics - suggestions for optimal algorithms. Journal of Computational Physics, 121(1):357-372, 1995. 
[2] L. Cullen and W. Dehnen. Inviscid smoothed particle hydrodynamics. Monthly Notices of the Royal Astronomical Society, 408(2):669-683, 2010.

[3] J. K. Dukowicz. A general, non-iterative riemann solver for Godunov's method. Journal of Computational Physics, 61(1):119 - 137, 1985.

[4] R. A. Gingold and J. J. Monaghan. Smoothed particle hydrodynamics: Theory and application to non-spherical stars. Monthly Notices of the Royal Astronomical Society, 181:375-389, 1977.

[5] S. K. Godunov. Difference methods for the numerical calculation of the equations of fluid dynamics. Math Sbornik, 47:221-306, 1959.

[6] L. Hernquist and N. Katz. TREESPH - a unification of SPH with the hierarchical tree method. Astrophysical Journal Supplement, 70:419-446, 1989.

[7] B. P. Howell and G. J. Ball. A free-lagrange augmented Godunov method for the simulation of elasticplastic solids. Journal of Computational Physics, 175(1):128-167, 2002.

[8] S.-I. Inutsuka. Reformulation of smoothed particle hydrodynamics with Riemann solver. Journal of Computational Physics, 179:238-267, 2002.

[9] G. R. Liu and M. B. Liu. Smoothed Particle Hydrodynamics, a meshfree particle method. World Scientific, 2003.

[10] J. J. Monaghan. Smoothed particle hydrodynamics. Reports on Progress in Physics, 68:1703-1759, 2005.

[11] J. J. Monaghan and R. A. Gingold. Shock simulation by the particle method SPH. Journal of Computational Physics, 52:374-389, 1983.

[12] G. Murante, S. Borgani, R. Brunino, and S.-H. Cha. Hydrodynamic simulations with the Godunov smoothed particle hydrodynamics. Monthly Notices of the Royal Astronomical Society, 417(1):136-153, 2011.

[13] A. N. Parshikov and S. A. Medin. Smoothed particle hydrodynamics using interparticle contact algorithms. Journal of Computational Physics, 180(1):358-382, 2002.

[14] J. I. Read and T. Hayfield. SPHS: Smoothed Particle Hydrodynamics with a higher order dissipation switch. Monthly Notices of the Royal Astronomical Society, 2011. In press.

[15] A. Shaw and S. R. Reid. Heuristic acceleration correction algorithm for use in SPH computations in impact mechanics. Computational Methods in Applied Mechanics and Engineering, 198(128):3962-3974, 2009.

[16] J. Swegle, J. Hicks, and S. Attaway. Smoothed particle hydrodynamics stability analysis. Journal of Computational Physics, 116:123-134, 1995.

[17] B. van Leer. Towards the ultimate conservative difference scheme - a second-order sequel to Godunov's method. Journal of Computational Physics, 32(1):101-136, 1979. 